\title{
Biomedical Applications of Fluorescence-Assisted Synchrotron Infrared Micro-Spectroscopy
}

\author{
LISA M. MILLER \\ National Synchrotron Light Source, Brookhaven National Laboratory, Upton, NY, USA
}

Infrared micro-spectroscopy (IRMS) is a widely used and valuable technique for examining the inherent chemical makeup of biological cells and tissues. With the advent of the synchrotron infrared microscope, new applications of IRMS are just beginning to be realized. This is largely due to the dramatic improvement in spatial resolution attainable using a synchrotron infrared source (Figure 1). The high brightness of the synchrotron source permits high quality data collection at the diffraction limit, e.g. 3-25 $\mu \mathrm{m}$ spatial resolution in the mid-infrared region $\left(4000-400 \mathrm{~cm}^{-1}\right)$. Thus, samples that are too small to be analyzed with an acceptable signal-to-noise using a conventional thermal (globar) source can now be examined, and the local chemical composition determined in greater detail. This is especially applicable to the biological and medical fields. For a typical biological specimen, the diffraction-limited spatial resolution for primary lipid (C-H stretch), protein (amide I), and nucleic acid (P-O stretch) absorption features is approximately 3,6 , and $12 \mu \mathrm{m}$, respectively.

With the ability to probe smaller and smaller areas with the synchrotron IR microscope, new techniques are currently being applied to aid in sample visualization. On the cellular and sub-cellular level, this technique can be used to visualize fluorescent tags bound to particular cellular components and even antibodies to individual proteins. Once identified, the IR microscope can be used to analyze the chemical environment in and around that region of interest. It should be noted that fluorescent labels are generally present in extremely low (i.e. nanomolar) concentrations, so they do not interfere with the IR technique; they are used exclusively for visualizing a region of interest. Figure 2 illustrates some examples of fluorescence visualization techniques that are particularly useful for examining biological specimens.

To date, combining fluorescence microscopy and infrared micro-spectroscopy has required analyses with two separate microscopes. This procedure required the user to somehow mark the fluorescent regions of interest (often with a photograph) before transferring the sample to the infrared microscope. The optical design of earlier infrared microscopes did not allow the ready insertion of accessory optics, such as optical components for fluorescence microscopy. In this talk, the development and use of a single microscope for simultaneous fluorescence microscopy and synchrotron IRMS will be described. Specific biomedical applications will be presented, including analysis of (1) bone tissue composition in osteoporosis, (2) misfolded protein structure in Alzheimer's disease, (3) tissue composition and tumor growth in cancer, and (4) cellular changes in apoptosis.

This work was performed at Beamline U10B at the National Synchrotron Light Source, Brookhaven National Laboratory. Collaborators on these projects include: Cathy Carlson (University of Minnesota); Mark Chance and Raymond Huang (Albert Einstein College of Medicine); David Burr (Indiana University School of Medicine); Judit Miklossy and Laszlo Forro (Swiss Institute of Pathology); Silvina Federman and Irit Sagi (Weizmann Institute); Paul Dumas, Nadege Jamin, and Jean-Luc Teillaud (LURE and Institute Curie-Paris). More information on the infrared programs at the NSLS can be found at hittp://infrared.nsis.bnl.govi.

*Corresponding Author Information: Lisa M. Miller, NSLS, Bldg. 725D, Brookhaven National Laboratory, Upton, NY 11973. Phone: (631) 344-2091; Fax: (631) 344-3238; Email: Imiller@bnl.gov 


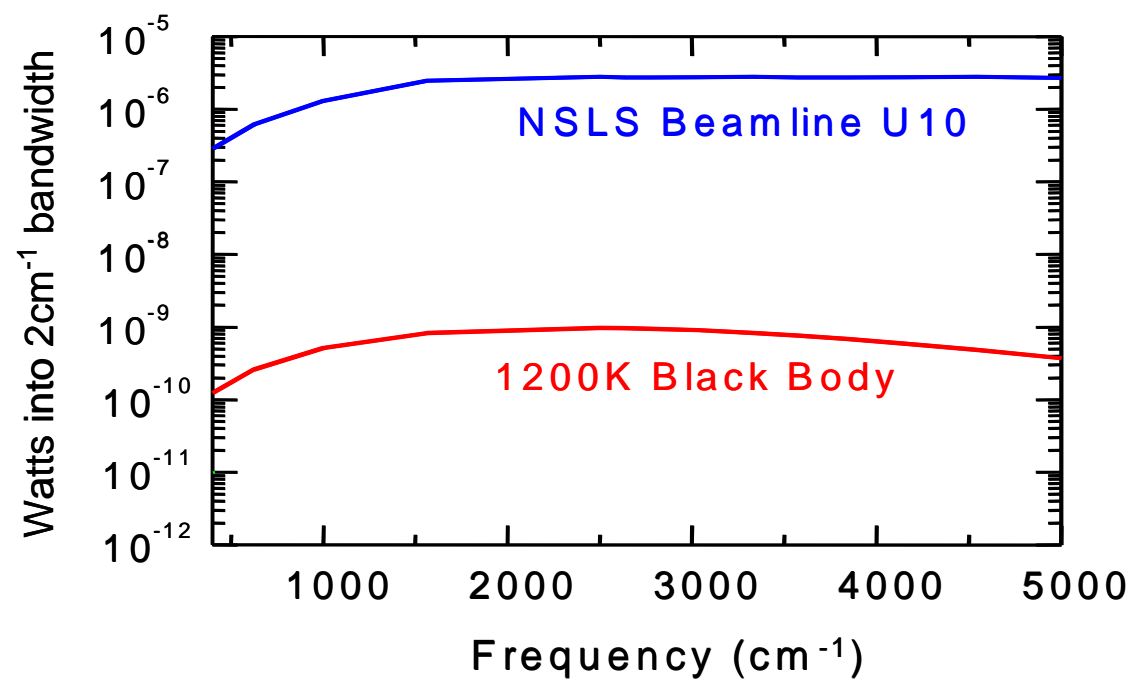

Figure 1. Synchrotron radiation is 2-3 orders of magnitude brighter than conventional globar sources as shown in this plot of the power delivered to a $10 \mu \mathrm{m}$ sample at $\mathrm{f} / 1$.

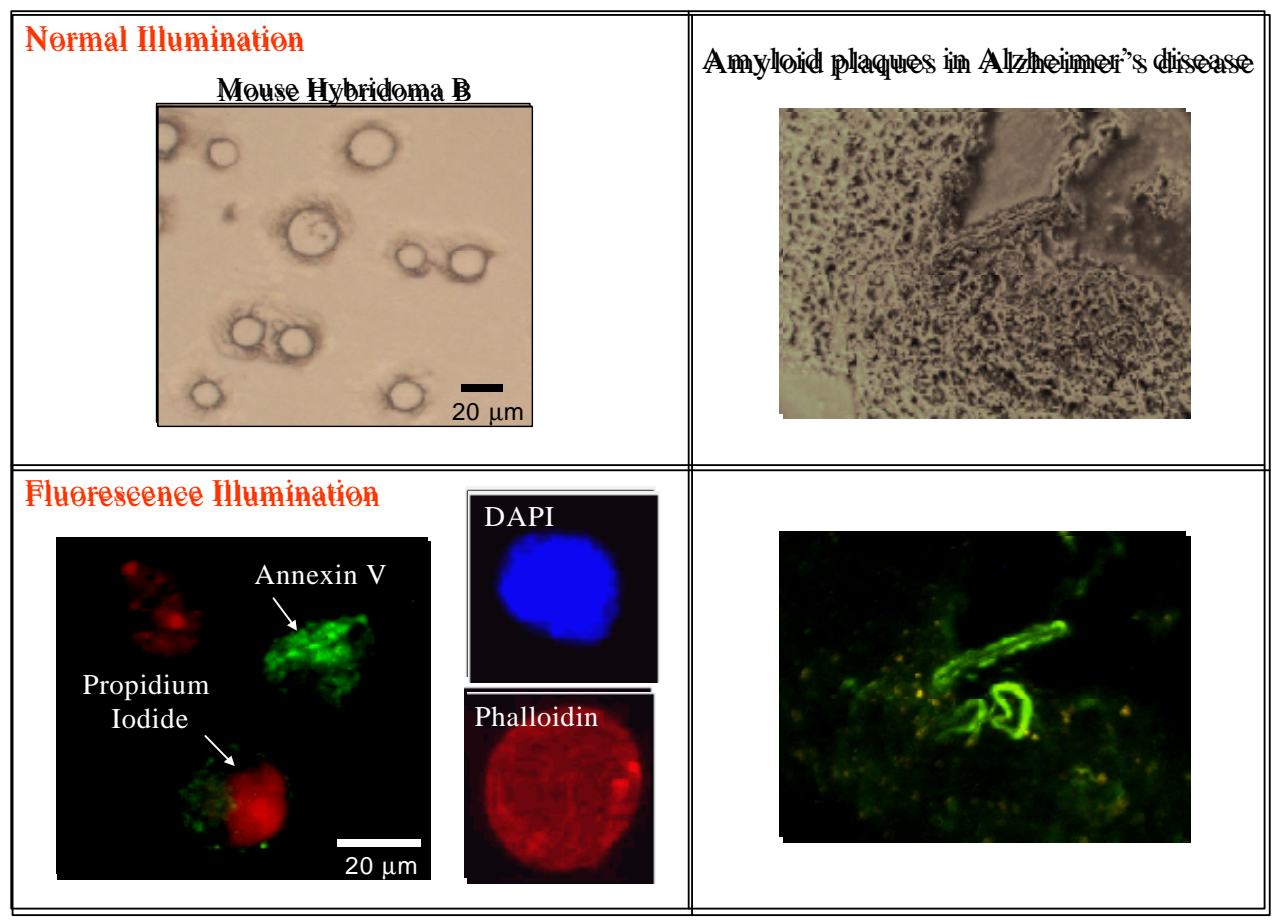

Figure 2. Normal (top) and fluorescence (bottom) illumination of mouse hybridoma $B$ cells (left) and amyloid plaques in Alzheimer's diseased brain (right). In the mouse cell, phalloidin is used to label the cytoplasm and DAPI is used to label the nucleus. During apoptosis, annexin $V$ and propidium iodide are used to identify early and late apoptosis, respectively. In the brain tissue, Thioflavin $S$ reveals amyloid plaques. 